\title{
Moulage in clinical simulation: step-by-step making of a third-degree burn
}

\begin{abstract}
Relevant components are necessary for high-fidelity simulation, including scripted scenarios, well-trained simulation educators and "actors," appropriate mannequins, and realistic environments. Moulage can be yet another key element when used properly. In health courses, moulage can be worked on using various themes. One of them worth working on is burn. This is considering that efficient care requires well-trained health professionals. The aim of this paper is to describe the preparation of moulage that represents a realistic, easy-to-perform third-degree burn using easily accessible and inexpensive materials. First, the researchers began with the search for specific literature, followed by the necessary material selection, and defining a model for the simulated burn. The moulage was executed in approximately 60 minutes. The step-by-step technique has been described and photographed, using low-cost, easily accessible materials to allow moulage to be considered and applied more often in the scenarios of various health-related areas. We believe our model can be used in a variety of simulation scenarios, making it relevant to educators and student learning.
\end{abstract}

Keywords: simulation, moulage, third-degree, burn
Volume 7 Issue 3 - 2020

\author{
Danielle R dos S Carvalho,' Patrícia da \\ S Klahr, ${ }^{2}$ Nícolas P Murcia, ${ }^{3}$ Melyssa L \\ Medeiros, ${ }^{4}$ Francisco GMRC Neto' \\ 'PhD in Biotechnology, Bionorte, State University of Amazonas, \\ Brazil \\ ${ }^{2}$ Master in Rehabilitation Sciences, Federal University of Health \\ Sciences of Porto Alegre, Brazil \\ ${ }^{3}$ Master in Health Sciences, Federal University of Rio Grande \\ Foundation, Brasi \\ ${ }^{4}$ Master in Biotechnology, Federal University of Rio Grande do \\ Norte, Brasil
}

\begin{abstract}
Correspondence: Patricia da Silva Klahr, Master in Rehabilitation Sciences, PhD student in Rehabilitation Sciences at the Federal University of Health Sciences of Porto Alegre (UFCSPA), Senior National Manager for Academic Quality of Health Sciences at Laureate, Academic Manager of UniFG and FG, Laureate, Brasil, Email patricia.klahr@gmail.com
\end{abstract}

Received: May II, 2020 | Published: May 28, 2020

\section{Introduction and background}

Although incipient in many countries, simulation as a strategy is gaining popularity in the context of training future health professionals. When associated with health, simulation is used to develop safe practice to reduce risks and improve the teaching and learning process. ${ }^{1,2}$ This is because simulation is a powerful tool used for skills training, allowing the learner to operate in a protected, safe, and controlled environment without the complications of real situations. $^{3}$

According to Guhde, ${ }^{4}$ using simulation as a teaching methodology has great advantages, such as helping undergraduate health students to build a more organized communication and develop greater confidence. While there is ample evidence that simulation-based training works, it is important to remember that when employed in an uncompromising and decontextualized manner, simulation can lose its potential to build on health excellence education. ${ }^{2}$ The relevant components to obtain a high-fidelity simulation include scripted scenarios, well-trained facilitators and "actors," appropriate mannequins, and realistic environments. ${ }^{5}$

Moulage is a term that currently refers to the application of special effects or make-up techniques to manikins and simulated or standardized patients. ${ }^{6}$ Moulages were used for educational purposes in the 19th century, serving as replicas of pathologic conditions. ${ }^{7}$ Moulage, when used appropriately, is another relevant component that can help bridge the gap between a clinical case and a simulation, making the scenario realistic.

In health education, simulation attempts to replicate essential aspects of a clinical situation, moulage uses materials such as makeup to make the student's experience more real. ${ }^{8}$ Moulage may be as simple as applying pre-made rubber or latex "wounds" to healthy patients' limbs, chest, hand, etc., or as complex as using complicated make-up and theater techniques to provide elements of realism to the training simulation. ${ }^{5}$ Therefore, moulage is very important and necessary to make the scenario more realistic and allow the student to immerse himself in the scene that is taking place, eliminating his disbelief. As the most common examples of moulage, we can mention bruises, wounds, burns, rashes, among others.

In health courses, moulage can be used in simulation scenarios and task trainings for courses such as Nursing, Physiotherapy, and Medicine. One of the topics worth working on is burn. This is because although there is no definite statistics on burns in Brazil, it is estimated that they exceed the US numbers of 40,000 hospitalizations per year due to burns, of which $10 \%$ result in death. ${ }^{9}$ In this context, we must consider that for efficient care, it is essential that health professionals are well-trained enough to quickly identify patient conditions and are able to care. ${ }^{10}$ There is a paucity of published literature pertaining to moulage, although the internet provides some interesting insights into how to use this technique. Thus, to contribute towards realistic simulations of health courses, the challenge and objective of this research was to describe how to prepare a moulage that represents a realistic, easy-to-perform third-degree burn using easily accessible, low-cost materials. The reasons for choosing a third-degree burn for this research was that these entail the highest morbidity and mortality among the different degrees of burn and that these are the kind of burns the student is less likely to perform, according to Queiroz et al. ${ }^{11}$

\section{Materials and methods}

For the development of moulage, the researchers began with the search for specific literature to obtain data and images that referred to the characteristics of a third-degree burn.The next step was the 
selection of material needed to perform the moulage representing the burn and after an initial experimentation, a model was defined for the simulated burn, suitable to demonstrate lesions in the face, arm, and pectoral regions. According to the literature, skin that has suffered a third-degree burn appears whitish/grayish, dry, hard, inelastic, and deformed. To obtain these characteristics and perform the technique, the following materials were used: $70^{\circ}$ alcohol (for site asepsis), modeling wax (white and red scar skin), varnish, latex, simulated blood, red, black, white, and red body paint; a stainless steel spatula, a make-up sponge, a liquid foundation matching the actor's skin tone. Arranging the set-up, the model and photographer, the separation of materials, and the execution of the moulage were done in a day, in September 2018.

\section{Results}

The following are the instructions for preparing the moulage and it took us approximately 60 minutes to prepare:

a. The working surface should be cleaned with $70 \%$ ethanol solution before starting the moulage.

b. A reasonable amount of varnish should be applied over the desired area to improve the adhesion of the modeling wax that will be applied next (Figure 1a).

c. The shape of the modeling wax (Scar white skin) that will represent the whitish and injured skin must be acquired using hands. Shape the modeling wax to suit the desired lesion (Figure 1b).

d. After shaping the modeling wax, it should be applied to the desired area, already varnished (Figure 1c).

e. The spatula should be used to press the modeling wax against the model's skin to unify the lesion area (Figure 1d).
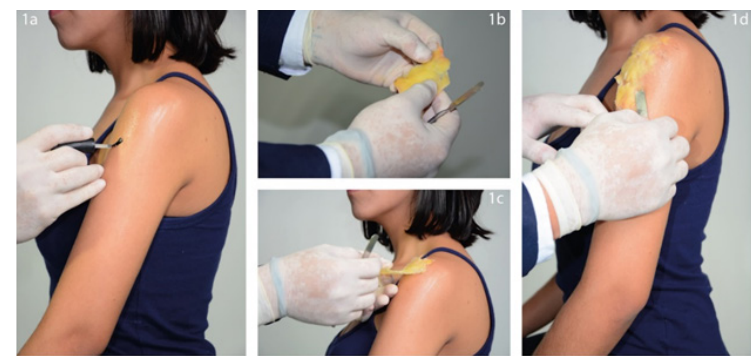

Figure I First stage of burn moulage production with application of varnish and modeling wax in the desired area.

f. With the help of a spatula shape the lesion, making an opening in the center to create depth (Figure 2a).

g. Liquid base must be applied to the edges of the lesion to unify the modeling wax on the model's skin (Figure 2b).

h. With the help of the make-up sponge spread the base until unifying the skin of the model (Figure 2c).

i. Black paint should be applied over the entire opening made into the modeling wax (white Scar skin) using the spatula to spread (Figure 2d).

j. It is necessary to apply black paint over and around the modeling wax (white Scar skin) for the effect of charred skin (Figure 3a). k. Simulated blood should be smeared using a make-up sponge on and around the lesion (as needed) (Figure 3b).

1. To extend the lesion, apply latex or white glue where wrinkled appearance is desired and spread well with using your finger (Figure 3c).

m. You should crumple a sheet of paper into a ball-like shape and then fully open the sheet to apply to the skin (Figure 3d).
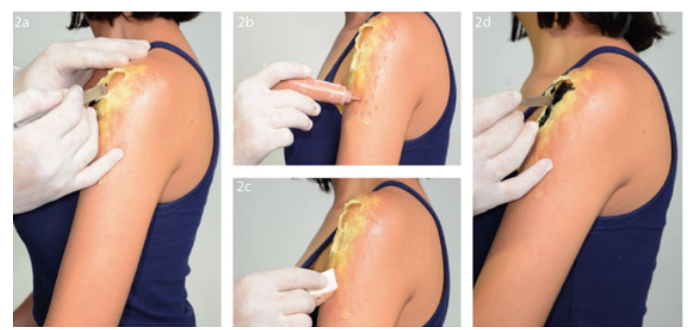

Figure 2 Manipulation of the modeling wax and application of liquid base and black paint.
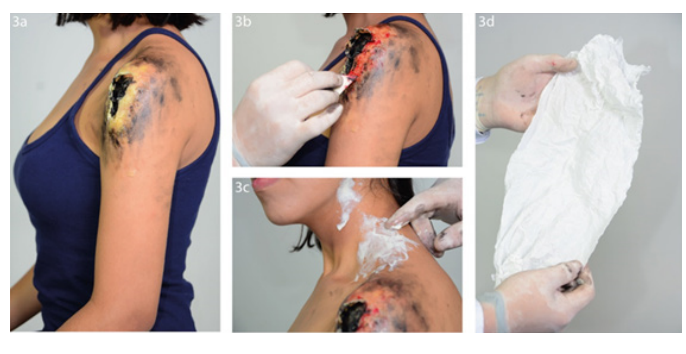

Figure 3 Application of black and red paint and latex. Preparation of paper to apply on site.

n. The sheet should be applied to the area with glue/latex. Remove excess paper not glued to the body (if necessary, apply glue to the side edges) (Figure 4a).

o. The modeling wax (white Scar Skin) should be used again and applied with the help of the spatula, respecting the extension of the desired lesion (making openings for depth effect) (Figure $4 b)$.

p. Black paint should be applied into the openings that were made in the modeling wax and around the lesions produced for the charred skin effect (Figure 4c).

q. Simulated blood or red paint should be applied on and around the lesion (as needed) (Figure 4d).
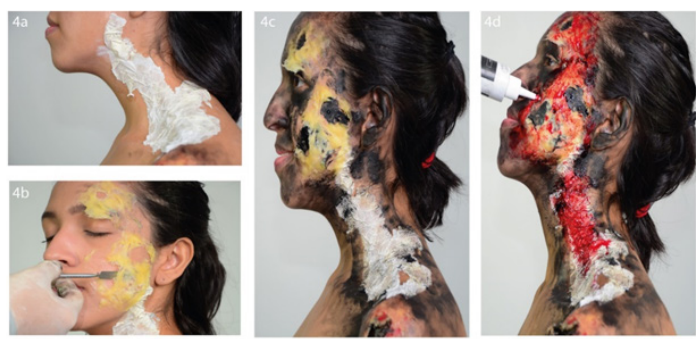

Figure 4 Application of paper and modelling wax to give the skin a wrinkled appearance. Spread black ink and simulated blood.

r. Spread simulated blood or red ink using your fingertip to unify the lesion (Figure 5a). 
s. With the make-up sponge, apply black paint around the lesion and over the sheet of paper glued to the skin to increase its extension and uniformity (Figure $5 b$ ).

t. Complete third degree burn moulage (Figure 5c).
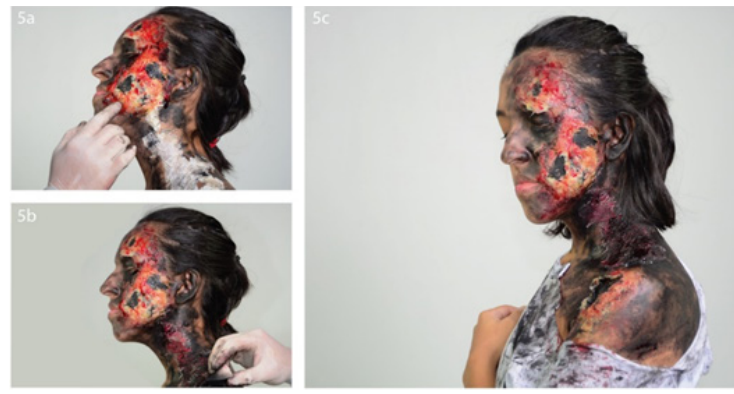

Figure 5 Finish by covering all paper and modelling wax with black ink and simulated blood. Burning moulage finished.

As noted, the step-by-step technique for simulating a third-degree burn was described and photographed to make it easy to perform even for a teacher without any experience of using the moulage technique in class. In addition, low-cost, easily accessible materials were used to allow moulage to be considered and applied more frequently in scenarios in various health areas. However, it should be noted that although we used easily accessible and inexpensive materials, care was always taken to ensure "fidelity," so that the end result of the moulage was as close as possible to a real burn.

\section{Discussion}

We know that during the simulation, students are exposed to different situations that may occur in a hospital or a community setting, providing them with opportunities to mobilize skills for customer appreciation, making decisions, communicating, working in a team, and managing the care of a simulated patient. ${ }^{12}$ However, a central question for everyone who uses simulation is how well a simulation replicates or represents "reality." The most prominent term to describe this reality relationship in simulation is "simulation fidelity." 13

"Fidelity" or "realism" is essential for the learning experience, as it allows students to feel that the experience was "real" and arouse in them the same psychological response as they would feel in a clinical setting. In other words, and we believe that by increasing the fidelity, we can increase the genuineness of the participants' actions and responses. ${ }^{12}$ With so many ways to improve fidelity in simulation, our purpose in this article was to enhance realism using moulage, as we believe this is an important and crucial strategy for the effectiveness of experiential learning.

Articles on moulage in general are few. Of these, a small number explore the idea that the moulage technique when associated with simulation could increase the retention of knowledge through its authenticity. ${ }^{14}$ What we do know is that probably because of the niche it fits, moulage may provide a unique opportunity to educate students. An example is that most articles that explore moulage at greater depth are in the field of Dermatology. ${ }^{15,16-19}$

The third-degree burn moulage was presented in this paper as an educational tool by proposing its association with a realistic simulation scenario. Moulage's primary role is to enhance visual realism, allowing the students to hone their clinical skills without risking patient safety. We believe that detailed technical descriptions, including the presentation of all necessary material used by our team to perform the moulage should be shared with the health simulation community. Since our model can be used in a variety of simulation scenarios, it is relevant to educators and student learning.We suggest, as a next step, to conduct studies that help us understand whether the use of simulation-associated moulage increases participant engagement and whether they are influenced by the authenticity of moulage.

\section{Acknowledgments}

We thank the models, artist photographer, and teachers who were part of this project, contributing through their expertise to explore the potential of moulage in health.

\section{Conflicts of interest}

There is no conflict of interest.

\section{Funding}

Authors had no funding for this research.

\section{References}

1. Jorge BM, Almeida RGS, Souza Junior VD. Trends in Research on Simulation in the Teaching of Nursing: An Integrative Review. Nursing Education Perspectives. 2014. p. 59-276.

2. Quilici AP, Abrão KC, Timermam S, et al. Clinical Simulation: from concept to applicability. São Paulo (SP): Editora Atheneu; 2012.

3. Beaubien JM, Baker DP. The use of simulation for training teamwork skills in health care: how low can you go? Qual Saf Health Care. 2004;13(1):i51-56

4. Guhde J. Using High Fidelity Simulation to Teach Nurse-to-Doctor Report: A Study on SBAR in an Undergraduate Nursing Curriculum. Clinical Simulation in Nursing. 2010;6(3):e115.

5. Kyle R. Technological resources for clinical simulation. In: Dunn WF, editor. Simulators in Critical Care and Beyond. Des Plaines, IL: Society of Critical Care Medicine. 2004. p. 95-113.

6. Smith-Stoner M. Using moulage to enhance educational instruction. Nurse Educ. 2011;36(1):21-24.

7. Lamb ME. The "Approximation Rule": Another Proposed Reform That Misses the Target. Child Dev Perspectives. 2007;1(2):135-136.

8. Pywella MJ, Evgeniou E, Highway K, et al. High fidelity, low cost moulage as a valid simulation tool to improve burns education. Burns. 2016;42(4):844-852.

9. Freitas MS, Machado MM, Moraes RZC, et al. Epidemiological characteristics of patients with third degree burns at the Hospital de Urgências de Sergipe. Rev Bras Burns. 2015;4(1):18-22.

10. Schweitzer IG, Nascimento ERP, Moreira AR, et al. Nursing care protocol in the aerospace environment for traumatized patients: pre-flight care. Rev Bras Enferm. 2011;64 (6):1056-1066.

11. Queiroz PR, Lima KC, Alcântara IC. Prevalence and factors associated with third degree burns in the city of Natal, RN - Brazil. Rev Bras Burns. 2013;12(3):169-176.

12. Foronda C, Liu S, Bauman EB. Evaluation of Simulation in Undergraduate Nurse Education: An Integrative Review. Clin Simul Nurs. 2013;9(10): e409-e416. 
13. Dieckmann P, Gaba D, Rall M. Deepening the Theoretical Foundations of Patient Simulation as Social Practice. Sim Healthcare. 2007;2:183-193.

14. Harder N. Dealing with the Fidelity of Simulation-Based Learning. Clinical Simulation in Nursing. 2018;25:20-21.

15. Bland AJ, Topping A, Tobbell J. Time to unravel the conceptual confusion of authenticity and fidelity and their contribution to learning within simulation-based nurse education. Nurse Educ Today. 2014;34(7):11121118.

16. Jain N, Anderson MJ, Patel P, et al. Melanoma simulation model: promoting opportunistic screening and patient counseling. JAMA Dermatol. 2013;149(6):710-716.
17. Hernandez C, Mermelstein R, Robinson JK, et al. Assessing students' ability to detect melanomas using standardized patients and moulage. $J$ Am Acad Dermatol. 2013;68(3):e83-e88.

18. Cooke RA. A moulage museum is not just a museum: wax models as teaching instruments. Virchows Archiv. 2010;457(5):513-520.

19. Kozlowski SWJ, DeShon RP. A psychological fidelity approach to simulation-based training: Theory, research, and principles. In: Salas E, Elliott LR, Schflett SG, editors. Scaled Worlds: Development, validation, and applications. Burlington, VT: Ashgate Publishing; 2004. p. 75-99. 